\title{
Quality Improvement in Critical Care: Patient-Focused
}

\section{Approach}

\author{
Tabish SA* \\ Professor, Sher-i-Kashmir Institute of Medical Sciences, India
}

*Corresponding author: Tabish SA, Professor, Sher-i-Kashmir Institute of Medical

Sciences, Srinagar, India; Email: amintabish@gmail.com; drtabish.sa@gmail.com

\section{Editorial}

Volume 4 Issue 1

Received Date: February 13, 2019

Published Date: February 21, 2019

\section{Editorial}

Medicines being an ever-changing science, health professionals are required to change their practice with broadening of our knowledge consequent to new research and clinical experience. Nearly everyone involved in the practice of Medicine is highly trained, is usually very well educated, and is committed to providing high quality of care. The ways in which quality is perceived, pursued, and insured continues to develop. Historically, much of what has driven the changes in healthcare has been the need for insuring quality across the entire healthcare system. The advancements in anesthesia techniques, availability of newer drugs, availability of newer advanced monitoring gadgets, increased awareness among the patient population, implementation of newer medicolegal laws and professional competitiveness has necessitated assurance of quality anesthesia services delivery. Scientifically sound methods for assessing quality exist and should be employed systematically in the future to guard against deterioration in quality that might otherwise occur as an unintended result of organizational and financial changes in the health services system. Quality is one of the major cornerstones of healthcare along with access to services and cost. Quality of Care is the degree to which Health Services for individuals and populations increase the likelihood of desired health outcomes and are consistent with current professional knowledge. Quality Assurance (QA) refers to "fit for purpose" and "do it right the first time". One of the most widely used paradigms for QA management is the PDCA (Plan-Do-Check-Act) approach, also known as the Shewhart cycle. The main goal of QA is to ensure that the product fulfills or exceeds customer expectations.
The quality approach places an emphasis on four aspects: infrastructure, elements such as controls, job management, adequate processes, performance and integrity criteria and identification of records, competence such as knowledge, skills, experience, qualifications, and soft elements, such as staff integrity, confidence, organizational culture, motivation, team spirit and quality relationships. The quality of the outputs is at risk if any of these four aspects are deficient in any way. The Institute of Medicine defines Quality of Care as the degree to which Health Services for individuals and populations increase the likelihood of desired health outcomes and are consistent with current professional knowledge. Total Quality Management (TQM) uses QA as its first step and seeks to implement the results of QA into a more comprehensive and continuous effort to improve Quality.

Quality improvement is a continuous effort by all the members of an organization to meet and exceed the needs and expectations of the patients and other customers. The goal is to not merely meet standards of care or to see them as limits to which we strive, but to exceed these standards. Performance assessment or measurement (QA) is a necessary step but it is not the end-it is the first step in a continuous cycle of improving quality (Continuous Quality Improvement: CQI).

Clinical Practice Guidelines and Standards of Care are systematically developed statements to assist practitioner and patient decisions about appropriate healthcare for specific clinical circumstances, rely on qualitative reasoning and emphasize clinical content, are written to influence practitioner behavior, are like expert opinion, 


\section{Anaesthesia \& Critical Care Medicine Journal}

require examination of evidence and values, must deal with the lack of best evidence, should include patient preferences, and should be graded according to strength of evidence in the overview, magnitude of effect and precision of the estimate.

While CQI focuses on industrial methods and TQM, on management philosophy, the terms often are used interchangeably because of their shared history and assumptions. Good quality should be designed into products and processes at the start, to prevent problems from ever arising. Inspections are important, to reject substandard products or services, but they cannot raise the quality of products or services produced.

Quality is achieved when: accessible services are provided in an efficient, cost-effective and acceptable manner that can be controlled by the ones providing it. QA is an organized process designed to ensure the maintenance of a desired level of safety and quality in a service or product. The aim is to continuously improve the safety and quality of care provided to patients and achieving excellent patient outcomes with the appreciation that expectations of acceptable outcomes should improve continuously, over time. There are many ways to assure or improve quality, but quality improvement should be systematic processes underpinned by science, based on scientifically plausible constructs grounded in first principles or empirical data, and supported by carefully selected measurements and sound analyses using suitable statistical methods when appropriate. Relevant expert advice is essential in designing projects and in the selection and interpretation of either quantitative or qualitative measures. QA initiatives include criteria-based audits. Selected aspects of structure, process or outcome are reviewed on a oneoff basis. Measures are evaluated against predetermined criteria. Clinical guidelines, policies, or protocols are an important part of QA, and their development, review and implementation should be linked to audit and ongoing measurement. The development of a policy can be an effective way of formulating consensus within a group on an important and potentially contentious issue.

Quality Improvement (QI) program should be designed with reference to all relevant domains of safety and quality taking into account local context. The development of capability and capacity is an important element of QI and provision should be made for participation of staff members in appropriate educational activities to increase their understanding and expertise in this field of knowledge. Risk management is an essential part of QA: it involves the regular, proactive identification of risks, assessment of risk factors, and implementation of controls to mitigate the identified risks. Mortality, and mortality and morbidity committees, supported by local reviews. Adverse reactions committees. Sentinel and serious adverse event reporting and root cause analysis: analysis of serious events associated with anaesthesia, perioperative care and pain management should be reported and reviewed with participation in institutional activities focusing on identification of contributory factors and the identification of actions to improve future safety. Critical incident reporting is well established at a binational level in anaesthesia. Participation in WEBAIRS should include local review of incident reports with a view to identifying and addressing contributing and mitigating factors. Strategies for improvement should be developed where possible, and actions taken to implement these strategies. Patient experience surveys are an important element of QI. These should focus on specific issues selected for example, patient's experience of pain as mild, moderate or severe, or how long it took for analgesia to be provided when requested.

Risk management is an important part of QA/QI: it involves the regular, proactive identification of risks, assessment of risk factors, and implementation of controls to mitigate the identified risks. Patient satisfaction is one of the most important results of goodquality care. Because patient satisfaction so influences their behavior, it is a critically important program goal. Patients often expect poor-quality care, accept it without complaint, and even express satisfaction when surveyed. Patient satisfaction is an important indicator of service quality. The principles of success include: strengthen systems and processes, encourage staff participation and teamwork. Base decisions on reliable information, improve communication and coordination and demonstrate leadership commitment. Systems thinking can help improve the delivery of family planning and other health care services. A systems perspective sees an organization as a collection of interdependent systems and processes. Human systems organize people. They include salaries and incentives, management and supervision, staff training and development. Technical systems are specific to the work of an organization, for example, the service delivery system in health care. Each system affects the other. Fixing flawed processes helps to improve staff performance.

A comprehensive quality control system uses different types of indicators, each measuring a different aspect of quality and providing complementary information. There are many ways to conceptualize and define indicators. Quality improvement (QI) is a revolutionary idea in health care. The aim is to raise the level of care through a 


\section{Anaesthesia \& Critical Care Medicine Journal}

continuous search for improvement. QI asks managers, providers, and other staff members to raise the norms. $\mathrm{QA} / \mathrm{QI}$ is a continuous, long-term process. Making quality a top priority requires fundamental changes in organizational culture, in goals and guidelines, and in daily operations. Organizations typically move forward in a series of small steps, each building on previous successes, rather than transforming themselves overnight. Persistence is crucial: Quality initiatives often must weather periods of discouragement, confusion, cynicism, and complacency before good quality can be achieved and maintained.

While CQI focuses on industrial methods and TQM, on management philosophy, the terms often are used interchangeably because of their shared history and assumptions. These approaches contend that good quality should be designed into products and processes at the start, to prevent problems from ever arising. Inspections are important, to reject substandard products or services, but they cannot raise the quality of products or services produced.

Quality design is a planning process. The design process defines the organization's mission, including its clients and services. It allocates resources and sets the standards for service delivery. Quality control consists of the monitoring, supervision, and evaluation that ensure every worker and every work unit meet those standards and consistently deliver good quality services. Quality improvement aims to increase quality and raise standards by continually solving problems and improving processes.

Good quality requires a patient-centered perspective that helps define quality and set program objectives and standards; a set of management principles that call for information-based, participatory, and collaborative decision-making and that focus on systems and processes to support and enable personnel; and a methodology to achieve, maintain, and advance good quality services by addressing all three points of the quality assurance triangle--quality design, quality control, and quality improvement. Quality management is a key concept in the health care system. It is essential to contribute to the collective assurance and improvement of the safety and quality of patient care by establishing that facilities or processes meet accepted standards. We have to promote QI to achieve increasingly better outcomes for patients. The process should progressively elevate the standards applicable for ongoing quality management. The objectives of QA/QI are improved quality, safety and experience of care for individual patients, improved health and equity for the population and best value for the available resources. High quality care implies: doing the right things (providing care that is evidence based and meets patients' individual needs and wishes) and doing things right the first time. The elements of quality in healthcare are: safety, timeliness, efficiency, efficacy, equitability, and patient-centredness. For high safety and quality care expertise and competence are critical which depends on training and on continuing professional development. To support and improve the standards of quality control, the data collection methods should be meticulous with effective feedback. Transparency, reliability, measurability, flexibility to improvement and above all a scientific platform are the essential parameters for serving as an effective quality indicator.

The process of measuring quality indicators and anaesthesia outcomes is highly challenging. Assessment of quality during perioperative period can be carried out by observing and recording any adverse event such as broken tooth, need for re-intubation and complications during difficult airway management, identification and management of cardiac and other complications related to co-morbid diseases, fluid overload and many others. Moreover, the quality can be assessed by identifying the timeliness of delivery of anaesthesia services or the frequency of line infiltrates.

Quality of the recovery care may be evaluated by assessing post-surgical complications such as hypotensive episodes, arrhythmias, respiratory complications, intakeoutput ratio, temperature fluctuations or causes for any prolonged stay in the recovery room.

The quality of anesthesia documentation and record keeping can be measured from the information recorded during the pre-anesthetic visit. This include drug history, history of systemic diseases including allergies, adverse drug reactions, addiction history, previous anesthesia experience, current medications and so on.

Methods of QI include voluntary incident reporting, multidisciplinary approach which requires a structured program with a shared goal between multidisciplinary participants within the clinical area, across clinical areas or across multiple health-care organizations to improve care, comprehensive unit-based safety programme, and quality improvement tools (daily goal sheets, briefings and debriefings and checklists).

Quality improvement efforts should include: Standardization of handoff processes and workflows whenever a change in anesthesia provider occurs, Standardization of operating room anesthesia tray set up and turnover processes between cases, Implementation of 


\section{Anaesthesia \& Critical Care Medicine Journal}

a standardized pre-procedure "time-out" prior to invasive procedures performed by the anesthesia care team, and Introduction of Emergency Manuals to provide guidelines that help clinicians with crisis management in specific situations of uncommon but life-threatening emergencies.

The Resident QA Programme should provide a forum to discuss patient safety events and suggestions for how they could improve the process and prevent similar events in the future.

Simulation training and facilitated debriefings are an important aspect of experiential learning for faculty and trainees. Putting patients first is key to improving the quality of health care. Organizations can design and offer services that both meet medical standards and treat patients as they want to be treated. Adopting a patientcentered approach often requires a shift in attitudes. An orientation to the patient recognizes that patients' concerns and preferences are important. In patientcentered health care, patients are considered first and foremost at every point in the planning, implementation, and evaluation of service delivery. Patients are the experts on their own personal circumstances and wants. What patients want is respect, understanding, complete and accurate information, technical competence, access, fairness and results. 